\title{
Study on the spatial roll angle measurement based on magneto- optic modulation
}

\author{
Wei Cai ${ }^{1,2}$, Zhiyong Yang ${ }^{1}$, Junhui Xing ${ }^{1}$ and Youan $\mathrm{Xu}^{1}$ \\ ${ }^{1} \mathrm{Xi}$ 'an High-Tech Institute, $710025 \mathrm{Xi}$ 'an, China \\ ${ }^{2}$ School of Telecommunication Engineering, Xidian University, $710071 \mathrm{Xi}$ 'an, China
}

\begin{abstract}
Faraday rotation angle formula for polarized light propagating in magneto-optic medium is investigated theoretically. Based on the Biot-Savart Law and Maxwell's equations, a calculation model for electromagnetic field in solenoid paraxial region is deduced. Taking advantage of this model, together with the Bessel functions, the axial magnetic field in a typical medium is calculated and analyzed. In addition, to thoroughly analyze the influence mechanisms of the incident wavelength and temperature on the Verdet constant and then uncover its essence, a hypothesis which suggests the Faraday effect results from the combination of various factors is proposed. Furthermore, a theory of wave-transition contribution to the Verdet constant is deduced by adopting the theory of wave-particle duality. Taking the typical diamagnetic material ZF1 and paramagnetic material TGG as examples, the deduced theory together with the corresponding models is tested and verified by analyzing the relevant parameters and the test data. At last, a testing system for magneto-optical rotation together with its finite element model is introduced.
\end{abstract}

\section{Introduction}

Spatial roll angle measurement based on magneto-optical modulation refers to measuring the roll angle between non-connected instruments in different horizontal planes, with polarized light and Faraday effect [1]. With the advantage of non-contact and high precision, this angle measurement technology has been gradually used in industrial, medical, biological, chemical detection and other fields, and it also has a broad application prospects in spacecraft docking.

Many works have been devoted to magneto-optical modulator signal generation, processing, and measurement system design, to improve the accuracy of roll angle measurement [2-5]. On the other hand, some researchers have studied the physical origin of the Faraday effect, and theories of the interactions among different physical fields in magneto-optical effect. Freiser derived the Faraday effect from Maxwell's equations and discussed heuristically its microscopic origin [6]. The research on its microscopic origin developed progressively and formed a sophisticated quantum theory $[7,8]$. However, the uncertain probabilities of electrons' distribution in different energy levels and of dipole transitions cause the usage of the quantum theory inconvenient. In order to describing the Faraday effect precisely and integrally, some efforts have been devoted to combine these two theories together in recent years $[9$, 10]. Besides the electromagnetic fields induced by the incident light and the applied magnetic field, the temperature around the magneto-optical material is another important factor which interacts with other physical fields and complicates the polarized light rotation. Liu researched the temperature dependence of Faraday effect in typical magneto-optical medium [11], and some practically applicable methods of compensating the temperature drift caused by the linear birefringence in Faraday material were developed [12].

The purpose of this paper is to analyze the factors which influence magneto-optical rotation. Following with a brief introduction of roll angle measurement principle in section 2, two important variables in Faraday rotation angle formula, magnetic field strength and Verdet constant, are studied and modelled theoretically in section 3 and section 4, respectively. In addition, a testing system for magneto-optical rotation together with its finite element model is presented in section 5 .

\section{Principle of roll angle measurement}

Fig. 1 schematically shows the principle of roll angle measurement based on magneto-optical modulation. A linear polarized light from the polarizer in upper instrument passes through the magneto-optical rotation glass in modulator, and its optical polarization plane will turn some angle because of the Faraday effect. The modulated signal received by analyzer in lower instrument is processed with lens, opto-electric conversion, amplification and certain algorithm. At the same time, the synchronous signal from upper instrument is transmitted to lower instrument, after that the roll angle is acquired. Various measurement models and corresponding algorithms have been developed $[1,5,13]$. 


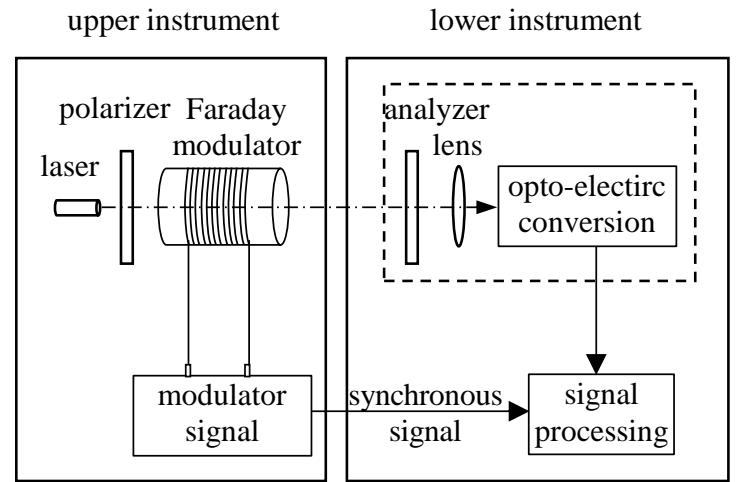

Fig 1. Principle of spatial roll angle measurement system based on magneto-optical modulation.

It can be proven that any linearly polarized wave can be seen as a superposition of two circularly polarized components, namely a left-circularly polarized (LCP) and a right-circularly polarized (RCP) one. The rotation of the light polarization plane, known as the Faraday rotation angle $\theta_{\mathrm{F}}$, is given

$$
\theta_{\mathrm{F}}=\pi L\left(n_{+}-n_{-}\right) / \lambda=V L B
$$

where $L$ is the length of magneto-optical medium, $n_{+}$ and $n$ - are the refractive indices for RCP light and LCP light, respectively, $\lambda$ is the light wavelength, $B$ is the strength of applied magnetic field, $V$ is the medium's Verdet constant. To acquire $\theta_{\mathrm{F}}$ accurately, the magnetic field in modulator, together with the medium's Verdet constant, which is dependant on light wavelength and temperature, should be modelled and calculated precisely.

\section{Magnetic field modelling in a solenoid with embedded magneto-optical medium}

Although different waveforms and multi-frequency wave compound are used in magneto-optic modulation [13], sine wave magneto-optical modulation is still the broadest used form, which is the base for studying other methods [14].

Based on the Biot-Savart Law, the magnetic flux density around a solenoid, together with its three components $\left(B_{\mathrm{z}}, B_{\mathrm{r}}, B_{\varphi}\right)$ in cylindrical coordinate, can be expressed as

$$
\begin{gathered}
\boldsymbol{B}(r, \varphi, z)=\frac{\mu_{0}}{4 \pi} \int_{s} \frac{\boldsymbol{J}_{s} \times \boldsymbol{R}}{D^{3}} \mathrm{~d} S \\
B_{z}=\frac{\mu_{0} n a I}{4 \pi} \int_{-\pi}^{\pi} \int_{-b}^{b} \frac{a-\cos \varphi}{D^{3}} \mathrm{~d} z d \varphi \\
B_{r}=\frac{\mu_{0} n a I}{4 \pi} \int_{-\pi}^{\pi} \int_{-b}^{b} \frac{(b-z) \cos \varphi}{D^{3}} \mathrm{~d} z \mathrm{~d} \varphi \\
B_{\varphi}=-\frac{\mu_{0} n I}{4 \pi} \int_{-b}^{b}(b-z)\left(\int_{-\pi}^{\pi} \frac{\sin \varphi}{D^{3}} \mathrm{~d} \varphi\right) \mathrm{d} z=0
\end{gathered}
$$

where $\boldsymbol{J}_{s}$ and $\boldsymbol{R}$ are the current density vector and radius vector, respectively, $a$ and $b$ are the radius and half length of the solenoid. Considering the solenoid coil stimulated by $I(t)=I_{m} e^{-\mathrm{i} \omega t}$, we get the conclusion that $B_{\varphi}$, $E_{\mathrm{z}}$ and $E_{\mathrm{r}}$ are equal to zero approximately. Furthermore, Maxwell's equations are adopted to study the relationship between the electric and magnetic fields, then we have

$$
\begin{gathered}
\frac{\partial E_{\varphi}}{\partial z}=-\mathrm{i} \omega B_{r} \\
\frac{1}{r} \frac{\partial\left(r E_{\varphi}\right)}{\partial r}=\mathrm{i} \omega B_{z} \\
\frac{\partial B_{r}}{\partial z}-\frac{\partial B_{z}}{\partial r}=-\mathrm{i} \omega \mu_{0} \varepsilon_{0} E_{\varphi}
\end{gathered}
$$

Through Eqs. (6) to (8), both the electric field and magnetic field around the solenoid can be work out.

Furthermore, the linear polarized light passes through the magneto-optical medium along the solenoid axis and forms a slender light beam, whose scope can be regarded as a paraxial region in which the interaction between the magnetic field and polarized light takes place. As well, we can get the conclusion that $B_{\mathrm{r}}$ is approximately equal to zero in the paraxial region. Hence, by combining Eqs. (6) to (8), we can acquire $B_{\mathrm{z}}$ and $E_{\varphi}$ as follow

$$
\begin{gathered}
r^{2} \frac{\partial^{2} B_{z}}{\partial r^{2}}+r \frac{\partial B_{z}}{\partial r}+k_{0}{ }^{2} r^{2} B_{z}=0 \\
r^{2} \frac{\partial^{2} E_{\varphi}}{\partial r^{2}}+r \frac{\partial E_{\varphi}}{\partial r}+\left(k_{0}{ }^{2} r^{2}-1\right) E_{\varphi}=0
\end{gathered}
$$

Eqs. (9) and (10) are the basic calculation models for the electromagnetic field in paraxial region of the solenoid stimulated by alternating current, in which $k_{0}=\omega \sqrt{\mu_{0} \varepsilon_{0}}$ is the wave vector in vacuum. For example, taking a typical medium without polarization relaxation into consideration, the axial magnetic component in (9) can be calculated by adopting the Bessel functions,

$$
B_{z}(r, t)=B_{0} \mathrm{~J}_{0}\left(k_{0} r\right) \cdot e^{-\mathrm{i} \omega t}
$$

where $B_{0}$ is the magnetic amplitude stimulated by direct current $I_{\mathrm{m}}$, and $\mathrm{J}_{0}\left(k_{0} r\right)$ is the zero order Bessel function.

Moreover, adopting (11) and keeping the solenoid length as a constant, the influence of solenoid radius on the amplitude of axial magnetic component $B_{z m}$ can be calculated, and its effect is shown in Fig. 2, in which the the solenoid length is $10 \mathrm{~cm}$, and the amplitude of the alternating current is $2 \mathrm{~A}$ with the coil density being 1000 turns $/ \mathrm{m}$.

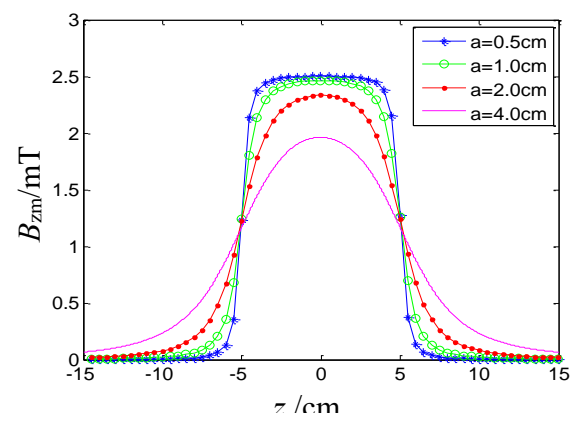

Fig 2. $B_{z m}$ in paraxial region with different radius $a$ 


\section{Verdet constant of magneto-optical materials}

In order to explore the influence mechanisms of the incident wavelength and temperature on the Verdet constant and then uncover its essence, two different theories are developed, including the classical electronic dynamics theory and quantum theory.

The former, based on the Lorentz dispersion theory, focuses on the oscillations of the electrons in magnetooptical materials under the combined influence of the polarized light and the applied magnetic field. Based on the classical electronic dynamics theory, Verdet constants for both the diamagnetic medium $V_{\text {dia }}$ and paramagnetic medium $V_{\text {par }}$ are obtained,

$$
\begin{gathered}
V_{d i a}=\frac{e \lambda}{2 m c} \frac{\mathrm{d} n}{\mathrm{~d} \lambda} \\
V_{p a r}=\frac{e \lambda}{2 m c} \frac{\mathrm{d} n}{\mathrm{~d} \lambda}(1+v \chi)
\end{gathered}
$$

in which $e$ and $m$ are the charge and mass of the electron, respectively, $c$ is the velocity of light in vacumm, $\mathrm{d} n / \mathrm{d} \lambda$ is the dispersion, $v$ is a coefficient in connection with Weiss field, $\chi$ is the magnetic susceptibility.

The latter, quantum theory, takes the differences of the probability and separation between ground and excited states, caused by applied magnetic field or medium magnetization, as the microcosmic essence of the Faraday rotation. Typical formulas for the paramagnetic medium $V_{\text {dia }}$ and diamagnetic medium $V_{\text {par }}$ can be described as

$$
\begin{aligned}
& V_{p a r}=\frac{4 \pi^{2} v^{2} \chi}{g \mu_{B} c h} \cdot \sum_{i j}\left[C_{i j} /\left(v^{2}-v_{i j}^{2}\right)\right] \\
& V_{d i a}=4 \pi N v^{2} \cdot \sum_{i j}\left[A_{i j} /\left(v^{2}-v_{i j}^{2}\right)^{2}\right]
\end{aligned}
$$

where $v$ is the frequency of incident light, $C_{i j}$ denotes the oscillator strengths of transitions with $v_{i j}, g$ is the Lande splitting factor, $\mu_{B}$ is the Bohr magneton number, $N$ is particles per volume, $A_{i j}$ denotes the transition moments.

However, neither of the two theories can be separately used to fully explain the Verdet constant and the correlative test data. Therefore, in order to thoroughly analyze the influence mechanisms of the incident wavelength and temperature on the Verdet constant and then uncover its essence, based on a hypothesis which suggests the Faraday effect results from the combination of various factors, we proposed a theory of wavetransition contribution to the Verdet constant by adopting the theory of wave-particle duality [10]. That is, the Faraday effect is caused by two different contributions simultaneously. One is the wave contribution, denoted as $\mathrm{Vl}$, which is the interaction between the wave aspect of light and the magneto-optical medium, and the other refers to the transition contribution, denoted as Vh, which comes from the electronic transition. When it comes to the deflection angle, the wave contribution is positive while the transition contribution is negative.
Based on the theory of wave-transition contribution, together with an approximation method, simplified formulas of the Verdet constant can be expressed as

$$
\begin{gathered}
V_{\text {dia }}=\frac{q_{e} \lambda}{2 m c} \frac{\mathrm{d} n}{\mathrm{~d} \lambda}-\frac{A \lambda_{t}^{4} \lambda^{2}}{\left(\lambda^{2}-\lambda_{t}^{2}\right)^{2}} \\
V_{p a r}=\frac{q_{e} \lambda}{2 m c} \frac{\mathrm{d} n}{\mathrm{~d} \lambda} \cdot\left(1+v \frac{T_{c}}{T-T_{p}}\right)-\frac{\lambda_{t}^{2}}{\lambda^{2}-\lambda_{t}^{2}} \frac{E}{T-T_{p}}
\end{gathered}
$$

Where $T_{c}$ is the Curie constant, $T_{p}$ is the paramagnetic Curie point, $T$ is the temperature, $\lambda_{t}$ and $C_{t}$ are the dominant transition wavelength and its corresponding probability, respectively, $q_{e}=-e<0, E=4 \pi^{2} C_{t} T_{c} /\left(g \mu_{B} c h\right)$ and $A=-4 \pi N A_{t} / c^{2}$ are coefficients independent with the wavelength $\lambda$.

Taking the typical diamagnetic material ZF1 and the typical paramagnetic material TGG as examples, the influences of the incident wavelength and the temperature on the Verdet constant are analyzed, and the deduced theory together with the corresponding models is tested and verified by analyzing the relevant parameters and the test data.

Fig. 3 and Fig. 4 show that the theoretical results correspond to the real values, which proves the rationality of the hypothesis and the authenticity of the deduced theory.

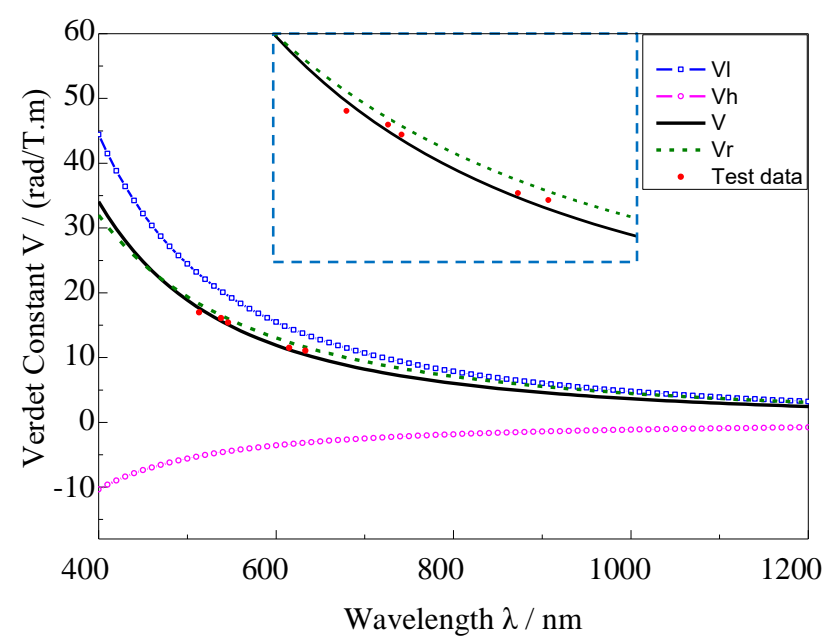

Fig 3. Wavelength dependence of Verdet constant of ZF1.

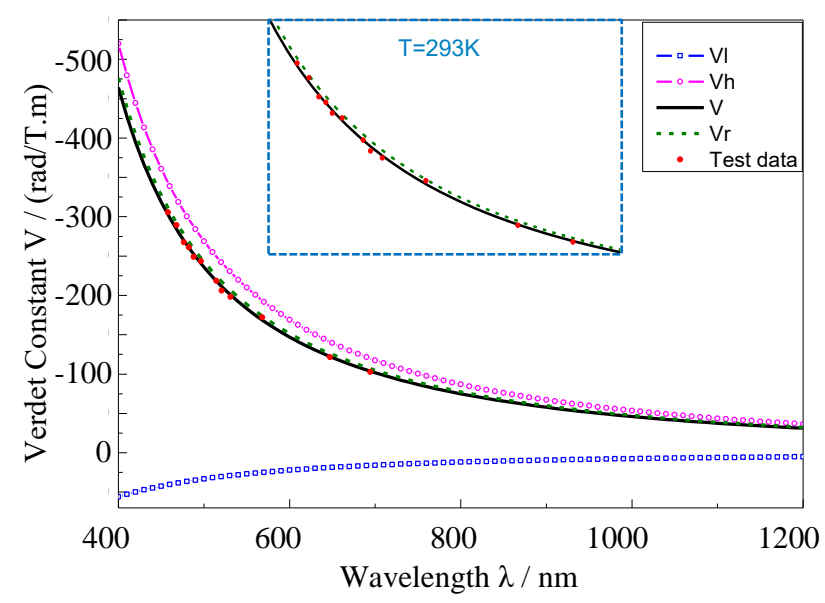

Fig 4. Wavelength dependence of Verdet constant of TGG $(\mathrm{T}=293 \mathrm{~K})$. 


\section{Testing system for magneto-optical rotation}

To measure the magneto-optical rotation angle's dependencies on both incident light wavelength and magnetic field strength, a testing system was designed as Fig. 5, in which both the incident light source and stimulating signal can be adjusted, and the magnetooptical glass in the modulator can be replaced with different magneto-optical materials as well.

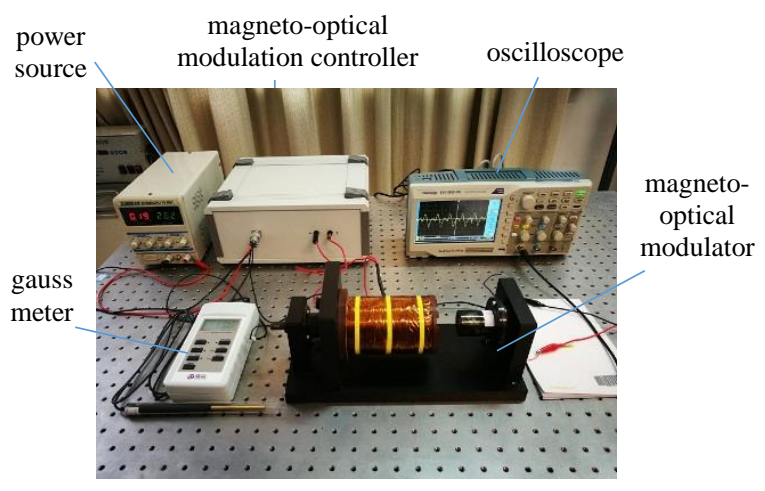

Fig 5. Testing system for magneto-optical rotation

The interaction between the polarized light and the applied magnetic field takes place in the magneto-optical glass surrounded by the solenoid coil, which is one of the key component in the magneto-optical modulator. In order to analyze the three-dimensional magnetic field stimulated by the solenoid coil visually, a finite element model of the modulator was built by using COMSOL software [14]. The geometric model and its mesh are shown in Fig. 6, and the solved magnetic field is given in Fig. 7.
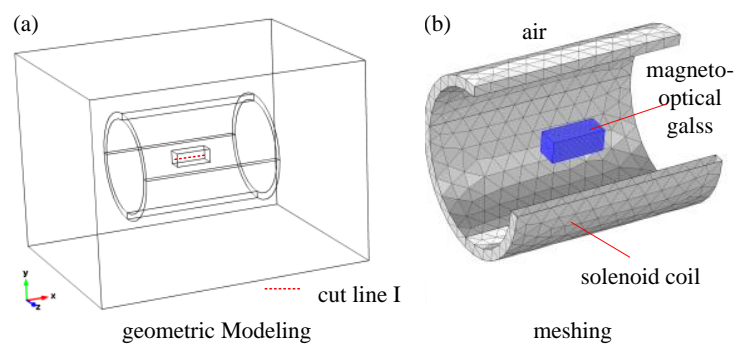

Fig 6. (a) Geometric model ; (b) mesh generation

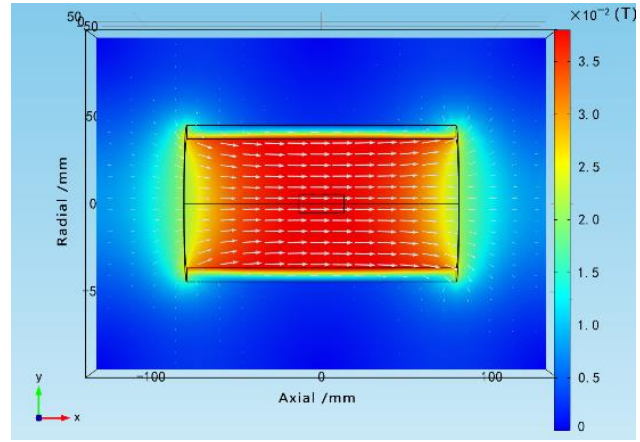

Fig 7. Magnetic field distribution arround the solenoid

\section{Conclusions}

The paper deals with two variables, $\mathrm{B}$ and $\mathrm{V}$, in the Faraday rotation angle formula. To the former, by adopting the Biot-Savart Law and Maxwell's equations, the electric field and magnetic field around the solenoid are analysed. Then, focusing on the solenoid axis in which the magneto-optical glass is set and the magneto-optical effect takes place, the calculation models for the electromagnetic field in paraxial region of the solenoid stimulated by alternating current are built, and the influence of solenoid radius on $B_{z m}$ is simulated numerically. To the latter, based on the essential property of the magneto-optical effect, a theory of wave-transition contribution to the Verdet constant is deduced by adopting the theory of wave-particle duality, and the deduced theory together with the corresponding models is tested and verified by analyzing the relevant parameters and the test data. The obtained results show that the simplified formulas of the Verdet constant for both diamagnetic and paramagnetic materials match well with the real values, and the proposed finite element model together with the magnetic field model gives a convenient approach to analysing physical fields in magneto-optical effect.

It should be noticed that either $\mathrm{B}$ or $\mathrm{V}$ is dependent on the temperature in solenoid, which is influenced by various of factors, and more serious, is time-varying. Therefore, both the temperature field in the working solenoid and its influence on $\mathrm{B}$ and $\mathrm{V}$ should be studied deeply in the future research, and multi-physical fields coupling analysis may be an effective method to address this challenge.

\section{ACKNOWLEDGEMENT}

The work presented in this paper was partially funded by the National Natural Sciences Foundation of China (grant No. 61505254) and the Aviation Science Foundation of China (grant No. 201551U8088).

\section{References}

1. X.N. Dong, L.M. Gao, X.J. Shen, L.Y. Chen, Acta Photonica Sinica 11, 1389-1391 (2001)

2. S.G. Li, C.X. Yang, E.Y. Zhang, G.F. Jin, Opt. Lett. 30 (3), 242-244 (2005)

3. P. Mihailovic, S. Petricevic, J. Radunovic, JINST 1 ( 12), 1-11 (2006)

4. S. Egami, H. Watarai, Rev. Sci. Instrum. 80 (9), 093705 (2009)

5. Z.Y. Yang, W.Cai, X.X. Huang, J.H. Xing, Optik 134, 194-202 (2017)

6. M. Freiser, IEEE Trans. on Mag. Mag-4 (2), 152-161 (1968)

7. F. Zhang, Y. Xu, J.H. Yang, M. Guillot, Eur. Phys. J. B 20, 165-175 (2001)

8. W. Wang, M. Gu, G. Liu, Phys. Rev. B 72, 014415 (2005)

9. T. Haider, Int. J. Electrom. \& App. 7 (1), 17-24 (2017) 
10. W. Cai, J.H. Xing, Z.Y. Yang, Acta Phys. Sinica 66 (18), 187801 (2017)

11. G.Q. Liu, W.K. Zhang, X. Zhang, Phys. Rev. B 48, 16091 (1993)

12. P. Menke, T. Bosselmann, J. Lightwave Technol. 13 (7), 1362-1370 (1995)
13. Z.Y. Yang, X.X. Huang, Z.F. Zhou, Z.L. Zhang, Optik 125 (3), 1042-1048 (2014)

14. Z.Y. Yang, X.X. Huang, Z.F. Zhou, Z.L. Zhang, Lasers in Eng. 26, 115-125 (2013) 